\title{
HLA-DR, HLA-DQ Haplotypes and Diabetic Autoantibodies in Nondiabetic Siblings of Type I Diabetes
}

Sitelbanat Awadalla ${ }^{1 *}$ and Manan AL Hakbany ${ }^{2}$

${ }^{1}$ Department of Basic clinical Sciences, College of Medicine, King Saud Bin Abdelaziz University, Riyadh, Saudi Arabia

${ }^{2}$ Department of Physiology, College of Medicine, King Saud University, Riyadh, Saudi Arabia

\begin{abstract}
The screening of non-diabetic siblings of Saudi type 1 diabetes mellitus (T1DM) patients ( $n=54)$ and 50 healthy controls was undertaken for glutamic acid decarboxylase (anti-GAD) and antibodies to tyrosine phosphatse (IA-2) using radioimmunoprecipitation. HLA-DRB1, DQB1 and DQA1 alleles were tested by PCR and sequence-specific oligonucleotide probes. HLA analysis showed that susceptible alleles were DRB1*03:01 (61.1\%) *04:01 (22.02\%), DQA1*05:01 (61.1\%), DQA1*03:03 (33.3\%) and DQB1*02:01 (72.2\%). The DRB1*03:01-DQA1*05:01-DQB1*02:01 haplotype was significantly higher among siblings $(61 \%)$. The protective alleles were DRB $1{ }^{*} 04: 03(1.8 \%)$, DRB $1 * 13$ (11.1\%), DQA1*02:01 (5.6\%), DQA1*05:05 (5.6\%), DQB1*03:01 (5.6\%) and DQB1*05:01 (11.1\%). GADA (22.2\%) and anti-IA-2 $(11.1 \%)$ were significantly higher among siblings, both antibodies present in $27.8 \%$ of siblings. The frequency of GADA was higher in those aged 5 to 10 years $(50 \%)$, while IA-2 positive children were > 5years old $(100 \%)$.

$36.4 \%$ of DRB1*03:01-DQA $1{ }^{*} 05: 01-D Q B 1 * 02: 01$ sibling were positive for GADA, $18.2 \%$ were positive for IA-2 and $9.1 \%$ were positive for both antibodies. $27.8 \%$ of siblings were HLA-identical to the proband, $61.1 \%$ were haploidentical, and $11.1 \%$ were not identical. GADA was significantly higher among the HLA-identical siblings $(60 \%)$ than haploidentical $(9.1 \%)$ and non-identical siblings (zero). IA-2 was higher in HLA-identical (20\%) from haploidentical $(9.1 \%$ ) and non-identical HLA (zero) but not to significant level. Both antibodies were present in $20 \%$ of HLA-identical siblings, and in none of the haplo- or non-identical HLA. In conclusion, the immunogenetic screening of nondiabetic sibling identifies individuals at risk of developing T1DM.
\end{abstract}

Keywords: GAD; IA-2; Type 1 Diabetes Mellitus; HLADR; DQ

\section{Introduction}

Over the last decade, the ultimate goal of diabetes research has focused on a better ability to predict T1DM so as to apply earlier intervention before the appearance of clinical symptoms [1]. Besides, the long subclinical prodromal period before the clinical manifestation of the disease gives an opportunity to identifying those individuals at risk [2]. Data obtained from research on the identification of high-risk individuals proved to be beneficial for intervention trials, and were vital clinically in counseling families with diabetic children [3].

Kulmala et al. [4] found that the analysis of high-risk genetic markers in combination with autoantibodies offers an excellent tool for identifying suitable at risk subjects to enroll in intervention trials. Decochez et al. [5] studied 4,589 Belgian first-degree siblings concluded that combined positivity for DQ2/DQ8 and IA-2 defined the highest risk population for prevention trials. On the other hand, Buczkowska and Chobot [6] suggested that autoantibodies alone in siblings were useful markers for identifying high risk individual for T1DM. Merna et al. [7] reported that, autoantibody status, susceptible alleles, and insulin sensitivity will be useful in assessing the risk of progression to type 1 diabetes in addition, to age and family history of type 1 diabetes at the time of diagnosis of the index.

A subsequent study included a large number of siblings of type I diabetic children $(n=701)$ who were followed for 15 years [8]. This study uncovered the strongest predictive model for progression to clinical disease which included age of sibling at the first sampling, HLA-DR that confer susceptibility, number of autoantibodies detected at the first sampling, and the number of affected siblings with Type 1 Diabetes Mellitus. The study also emphasized the role of IA-2 in the prediction of Type I Diabetes [8]. Numerous other studies have explored the association of DRB1, DQA1, and DQB1 with type 1 diabetes in different ethnic background [9-12]. However, the current study is the first to be undertaken in Saudi Arabia.

Thus the aim of this study is to test Saudi non-diabetic siblings of T1DM and controls for HLA DRB1, DQA1 and DQB1 haplotypes and test them for the presence of anti-GAD65 and anti-IA-2 autoantibodies.

\section{Materials and Methods}

\section{Siblings and controls}

The studied population composed of 54 non-diabetic sibling of Saudi TIDM. 26 were females and 28 males. Their mean age was 7.5 years at the time of proband diagnosis (range 1-20 years). Healthy controls $(n=50)$ had no family history of T1DM. All particpants were screened for HLA-DRB1, HLA-DQA1, HLA-DQB1 and for autoantibodies against GAD and IA-2. Further, participants or their parents gave informed written consent, and the study was approved by the College of Medicine Institutional Research Board (IRB).

*Corresponding author: Dr. Sitelbanat A Awadalla, Department of Basic Medical Science, College of Medicine, King Saud Bin Abdel Aziz University, National Guard, P.O. Box 22490, Riyadh 11426, Saudi Arabia, Tel: +9661 4291341; E-mail: sitelbanat33@gmail.com

Received May 16, 2015; Accepted June 29, 2015; Published July 03, 2015

Citation: Awadalla S, AL Hakbany M (2015) HLA-DR, HLA-DQ Haplotypes and Diabetic Autoantibodies in Nondiabetic Siblings of Type I Diabetes. J Diabetes Metab 6: 580. doi:10.4172/2155-6156.1000580

Copyright: (c) 2015 Awadalla S, et al. This is an open-access article distributed under the terms of the Creative Commons Attribution License, which permits unrestricted use, distribution, and reproduction in any medium, provided the original author and source are credited. 
Citation: Awadalla S, AL Hakbany M (2015) HLA-DR, HLA-DQ Haplotypes and Diabetic Autoantibodies in Nondiabetic Siblings of Type I Diabetes. J Diabetes Metab 6: 580. doi:10.4172/2155-6156.1000580

Page 2 of 5

\section{Laboratory assays}

HLA genotyping: Total genomic DNA was extracted from the peripheral blood mononuclear leukocytes from siblings and controls using Qiagen Kit (27220 Turnberry Lane, Valencia CA 91355). The HLA-DRB1, DQB1 and DQA1 gene alleles were analyzed by the PCRsequence-specific priming technique, using the Micro SSP ${ }^{\text {ma }}$ DNA Typing Trays (one lambda, Inc. 21001 Kittridge Street Canoga Park, CA 91303-2801 USA). The sequence-specific oligonucleotide primers used for the amplification of HLA alleles and the human B-globin gene were used as negative controls. Pre optimized primers were presented (dried) in different wells of a 96-well $0.2 \mathrm{ml}$ thin-walled tube tray to be used for PCR testing and ready for the addition of DNA samples, recombinant Taq polymerase, and specially formulated dNTP-buffer mix (Micro SSP ${ }^{\mathrm{ma}}$ D-mix, one lambda). Each tray included a negative control reaction tube that detected the presence of the internal control PCR product generated by the Micro SSP ${ }^{m x}$ DNA Typing Tray. The PCR products were analyzed on a $2.5 \%(\mathrm{wt} / \mathrm{vol})$ agarose gel stained with ethidium bromide $(0.5 \mu \mathrm{g} / \mathrm{ml})$.

Autoantibodies assays: The antibodies against IA-2 and GAD were quantified using the radio binding assay as described previously [13]. Serum samples were incubated with the antigen in a 1:25 dilution in duplicates, and precipitated with Protein A-Sepharose (Amersham Biosciences, Uppsala, Sweden). The plates were counted in a Microbeta counter, and the results expressed as levels of antibodies calculated in relation to a standard curve. The cut-off limit for positivity corresponding to the 98th percentile of 50 healthy controls was 5.35RU for GAD and 0.43 for IA-2. The specificity of GADA assay was $99 \%$ and for IA-2 was $97 \%$, while the sensitivity was $75 \%$ for GADA and $60 \%$ for IA-2. Inter-assay variation for negative and positive controls was $8 \%$ and $14 \%$ for GADA and $13 \%$ and $11 \%$ for IA-2.

\section{Data analysis}

HLA haplotype frequencies were calculated by direct gene counting. The statical difference in the HLA haplotype distributions between the siblings and controls were determined using Chi-square test $\left(\mathrm{X}^{2}\right)$ test with Yates' correction (two-tailed). The odds ratio (OR) and $95 \%$ confidence interval were calculated using the Woolf formula to assess the susceptibility effect of the HLA DRB1 and DQB1 alleles and haplotype [14]. The Fisher's Exact Test was used when the expected frequency for the haplotype was less than five. A p of value $<0.05$ was considered statistically significant. Correlation between DRB1, DQA1 and DQB1 frequencies and age at the onset were analyzed using the Chi-square test. Statistical analysis was performed using SPSS 22.1 for Windows (SPSS, Chicago, IL).

\section{Results}

\section{HLA frequencies among siblings of T1DM patients and} controls

HLA-DR: The commonest allele among siblings was $\mathrm{DRB1}^{\star} 03: 01$ (Table 1). The frequency of $\mathrm{DRB} 1^{\star} 03: 01(61.1 \%)$ was significantly higher in siblings compared to controls (16\%) (X 20.27, P=0.0001, $\mathrm{OR}=8.25,95 \% \mathrm{CI} 2.98-23.56)$. $\mathrm{DRB} 1^{\star} 04: 01$ was significantly higher in siblings (22.1\%) than controls (2\%) (X7.95, p=0.005, OR14, 95\% CL $1.75-2.708)$. The DRB1*04:03 (1.8\% versus $16 \%)$ and DRB1*13 $(11.1 \%$ versus $34 \%)$ were significantly lower among siblings than controls (X 3.9, $\mathrm{p}=0.04$, OR 0.12, 95\% CI 0.005-0.989), (X 6.6, p 0.01, OR 0.243, $95 \%$ CI 0.076-0.747) respectively.

The DRB1 ${ }^{\star}$ 03:01 homozygote haplotype was significantly higher in siblings $(22.2 \%)$ compared to controls $(2 \%)$ (X 7.95, p 0.005, OR 14.0, 95\% CI 1.76-300.26). The DRB1*03:01-04:05 haplotype was significantly higher in siblings (14.8\%) compared to controls (2\%), (X 3.89, p 0.04, OR 8.5, 95\% CI 1.0-188.79).

DQA1: The DQA1*03:03 (33.3\%versus 6\%) and DQA1*05:01 (61.1\% versus $26 \%)$ were significantly higher in siblings than controls (X 10.4, $\mathrm{p}=0.001$, OR 7.83 95\% CL 1.96-36.44), (X11.59 p=0.001, OR $4.47,95 \%$ CL $1.95-10.24)$ respectively (Table 2$)$. The DQA1*02:01 (5.6\% versus $54 \%)$ and $\mathrm{DQA1} 1^{\star} 05: 05(5.6 \%$ versus $14 \%)$ were significantly lower in siblings than controls (X 27.37, $\mathrm{p}=0.0001$, OR $0.05,95 \% \mathrm{CL}$ 0.2-0.17), (X 11.75, p=0.001, OR 0.11, 95\% CL 0.03-0.39) respectively.

DQB1: The DQB ${ }^{*} 02: 01$ was significantly higher in siblings (72.2\%) than controls (30\%). (X16.8, p=0.0001, OR 6.06, 95\%CL 2.614.08). The DQB1 ${ }^{\star} 03: 01$ (5.6\% versus $\left.26 \%\right)$ and $\mathrm{DQB} 1^{\star} 05: 01(11.1 \%$ versus $36 \%$ ) were significantly lower in siblings compared to controls (X6.83, $\mathrm{p}=0.009$, OR 016, 95\%CL 0.05-0.59), (X7.7, $\mathrm{p}=0.005$ OR 0.22 , 95\% CL 0.08-0.62) respectively.

The homozygote $\mathrm{DQB1}{ }^{\star} 02: 01$ (33.3\%) was significantly higher in siblings than controls (12\%) (X5.51, p=0.019, OR3.67, 95\% CL 1.2 11.65). The $\mathrm{DQA} 1{ }^{*} 05: 01-\mathrm{DQB} 1^{*} 0201$ haplotype was also significantly higher among sibling (61\%) compared to controls (26.7\%) (X11.6, $\mathrm{p}=0.001$, OR $4.47,95 \%$ CL $1.9-10.2$ )

\begin{tabular}{|c|c|c|c|c|c|c|}
\hline DRB1 allele & $\begin{array}{cc}\operatorname{Siblings}_{(\%)} & n=54\end{array}$ & $\begin{array}{l}\text { Controls } \\
n=50(\%)\end{array}$ & $\mathbf{X}^{2}$ & $P$ value & OR & $\mathrm{Cl}$ \\
\hline $\mathrm{DRB} 1 * 01$ & $6(11.1)$ & $10(20)$ & 0.97 & 0.33 & 0.5 & $0.38-8.12$ \\
\hline DRB1*03 & $33(61.1)$ & $8(16)$ & 20.27 & 0.0001 & 8.25 & $2.98-23.56$ \\
\hline $\mathrm{DRB}^{*} 04$ & 25 (46.29) & $17(34)$ & 1.16 & 0.28 & 1.67 & $0.65-3.72$ \\
\hline *04:01 & $12(22.2)$ & $1(2)$ & 7.95 & 0.005 & 14 & $1.75-2.708$ \\
\hline${ }^{*} 04: 02$ & $1(1.8)$ & $2(4)$ & 0.05 & 0.95 & 0.45 & $0.02-2.77$ \\
\hline *04:03 & $1(1.8)$ & $8(16)$ & 3.9 & 0.04 & 0.12 & $0.005-0.989$ \\
\hline *04:04 & $2(3.7)$ & $1(2)$ & 0.00 & 1.00 & 1.07 & $0.186^{*} 6.03$ \\
\hline *04:05 & $8(11.1)$ & $1(2)$ & 2.36 & 0.12 & 4.17 & $0.76-30.18$ \\
\hline${ }^{*} 04: 06$ & $1(1.85)$ & $3(6)$ & 4.6 & 0.03 & 0.17 & $0.03-0.87$ \\
\hline DRB1*13 & $6(11.1)$ & $17(34)$ & 6.6 & 0.01 & 0.243 & $0.076-0.747$ \\
\hline DRB1*14 & $1(1.8)$ & $4(7.4)$ & 1.0 & 0.32 & 0.22 & $0.03-1.50$ \\
\hline DRB1*15 & $15(27.8)$ & $10(20)$ & 0.49 & 0.48 & 1.54 & $0.63-3.77$ \\
\hline DRB1*03:01/03:01 & $12(22.2)$ & $1(2)$ & 7.95 & 0.005 & 14.0 & $1.76-300.26$ \\
\hline DRB1*03:01/04:05 & $8(14.8)$ & $1(2)$ & 3.89 & 0.04 & 8.5 & $1.0-188.79$ \\
\hline
\end{tabular}

Table 1: DRB1 distribution among Siblings of T1DM and Control Subjects. 


\begin{tabular}{|c|c|c|c|c|c|c|}
\hline DQA1 & $\begin{array}{l}\text { Siblings } \\
n=54(\%)\end{array}$ & $\begin{array}{l}\text { Controls } \\
n=50(\%)\end{array}$ & $\mathbf{X}^{2}$ & P Values & OR & $95 \% \mathrm{Cl}$ \\
\hline${ }^{*} 01: 01$ & $6(11.1)$ & $2(4)$ & 0.98 & 0.32 & 3.0 & $0.6-13.5$ \\
\hline${ }^{*} 01: 02$ & $18(33.3)$ & $13(26)$ & 0.36 & 0.54 & 1.4 & $0.62-3.2$ \\
\hline *01:03 & $3(5.6)$ & $8(16)$ & 1.99 & 0.16 & 0.31 & $0.08-1.15$ \\
\hline${ }^{*} 01: 05$ & $1(1.8)$ & $2(4)$ & 0.005 & 0.95 & 0.45 & $0.05-3.59$ \\
\hline *02:01 & $3(5.6)$ & $27(54)$ & 27.37 & 0.0001 & 0.05 & $0.2-0.17$ \\
\hline${ }^{*} 03: 01$ & $9(16.7)$ & $12(24)$ & 0.47 & 0.45 & 0.63 & $0.24-1.6$ \\
\hline${ }^{*} 03: 03$ & $18(33.3)$ & $3(6)$ & 10.40 & 0.001 & 7.83 & $1.96-36.44$ \\
\hline *04:01 & $3(5.6)$ & $1(2)$ & 0.19 & 0.66 & 2.88 & $0.39-20.7$ \\
\hline *05:01 & 33 (61.1) & $13(26)$ & 11.59 & 0.001 & 4.47 & $1.95-10.24$ \\
\hline *05:05 & $3(5.6)$ & $7(14)$ & 11.75 & 0.001 & 0.11 & $0.03-0.39$ \\
\hline \multicolumn{7}{|l|}{ DQB1 } \\
\hline *02:01 & $39(72.2)$ & $15(30)$ & 16.8 & 0.0001 & 6.06 & $2.6-14.08$ \\
\hline${ }^{*} 03: 01(7)$ & $3(5.6)$ & $13(26)$ & 6.83 & 0.009 & 0.16 & $0.05-0.59$ \\
\hline${ }^{*} 03: 02$ (8) & $12(22.2)$ & $10(20)$ & 0.001 & 0.971 & 1.14 & $0.45-2.88$ \\
\hline${ }^{*} 04: 02$ & $9(16.7)$ & $5(10)$ & 0.51 & 0.47 & 1.8 & $0.58-5.5$ \\
\hline *05:01 & $6(11.1)$ & $18(36)$ & 7.7 & 0.005 & 0.22 & $0.08-0.62$ \\
\hline *06:02 & $21(38.9)$ & $23(46)$ & 0.28 & 0.59 & 0.74 & $0.34-1.62$ \\
\hline $\begin{array}{l}\text { DQB1*02:01/ } \\
\text { DQB1*02:01 }\end{array}$ & $18(33.3)$ & $6(12)$ & 5.51 & 0.019 & 3.67 & $1.2-11.65$ \\
\hline $\begin{array}{l}\text { DRB1*03:01- } \\
\text { DQA1*05:01- } \\
\text { DQB1*02:01 }\end{array}$ & $33(61)$ & $13(26.7)$ & 11.6 & 0.001 & 4.47 & $1.9-10.2$ \\
\hline
\end{tabular}

Table 2: The frequency of HLA-DQA1, DQB1 haplotype among siblings of T1DM and controls.

\begin{tabular}{|c|c|c|c|c|c|c|c|}
\hline & $\begin{array}{c}\text { HLA-identical } \\
15(27.8 \%)\end{array}$ & $\begin{array}{l}\text { HLA-haplo- } \\
\text { identical } \\
33(61.1 \%)\end{array}$ & $\begin{array}{c}\text { HLA-un identical } \\
6 \\
(11.1 \%)\end{array}$ & $\mathbf{X}^{2}$ & Pc value & OR & $95 \% \mathrm{Cl}$ \\
\hline GADA+ve & $9(60 \%)^{*}$ & $3(9.1 \%)$ & 0 & 11.66 & 0.001 & 15 & $3.3-67.8$ \\
\hline IA-2+ve & $3(20 \%)$ & $3(9.1 \%)$ & 0 & 6.34 & 0.55 & 2.5 & $0.5-12.6$ \\
\hline $\begin{array}{l}\text { GAD+ve \& } \\
\text { IA-2 +ve }\end{array}$ & $3(20 \%)^{*}$ & 0 & 0 & 7.04 & 0.008 & $\inf$ & 1.9-inf \\
\hline
\end{tabular}

$\mathrm{P}<0.05$ comparing HLA identical with HLA haploidentical

Table 3: The relation between HLA sharing with affected proband and autoantibodies in siblings of T1DM patients.

\section{The genotype of siblings of T1DM patients}

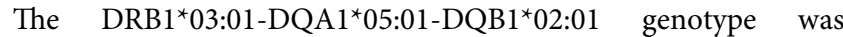
significantly higher in siblings $(61.1 \%)$ compared to controls $(26 \%)$ (X 11.89, $\mathrm{p}=0.001, \mathrm{OR}=4.47 ; 95 \% \mathrm{CL} 1.79-11.31$ ). The homozygote $\mathrm{DRB} 1^{\star} 03: 01-\mathrm{DQA} 1^{\star} 05: 01-\mathrm{DQB} 1^{\star} 02: 01$ was significantly higher in siblings $(22.2 \%)$ compare to controls $(2 \%)$ (X 7.95, $\mathrm{P}=0.005 ; \mathrm{OR}=14$, 95\% CL 1.76-300.2).

\section{The frequency of anti-GAD and anti-IA-2 among Sibling of T1DM patients}

Twelve siblings were positive for anti-GAD (22.2\%) significantly higher compared controls (zero) $(\mathrm{p}=0.001)$. Six were positive for antiIA-2 $(11.1 \%)(\mathrm{p}=0.015)$ significantly higher compared to none of the controls. Three siblings (5.6\%) were positive for both GADA and IA-2 antibodies (Table 3). The frequency of GADA was higher in the age group 5 to 10 years (50\%), while all IA-2 positive were under the age of 5 years $(100 \%)$

\section{Antibodies correlation with HLA sharing}

$27.8 \%$ of siblings were HLA-identical to the proband, $61.1 \%$ were haploidentical and $11.1 \%$ were nonidentical. GADA frequency was significantly higher $(60 \%)$ among the HLA-identical siblings than among the haplo $(9.1 \%)$ or non-identical siblings (zero) $(\mathrm{p}=0.001)$.
The frequency of IA-2 was higher in HLA-identical (20\%) compared to haploidentical (9.1\%) and nonidentical (zero) (Table 3). For multiple antibodies, the presence of two antibodies was significantly higher among HLA-identical siblings $(20 \%)(\mathrm{p}=0.008)$ than haplo and nonidentical siblings (zero). $36.4 \%$ of siblings with DRB1 ${ }^{\star} 03: 01-$ $\mathrm{DQA}^{\star}{ }^{*} 05: 01-\mathrm{DQB} 1{ }^{\star} 02: 01$ were positive for GADA, $18.2 \%$ were positive for IA-2 and $9.1 \%$ were positive for both antibodies.

\section{Discussion}

The aim of this study was to screen non-diabetic siblings of type 1 diabetic Saudi for HLA gene and autoantibodies at the time of proband diagnosis. This study meant to provide a genetic and immunological basis for understanding the mechanisms leading to islet beta-cell destruction among nondiabetic sibling in Saudi population. It is also allowing us to establish markers to identify high-risk individuals for the intervention trials. Genetic studying of siblings of T1DM gives vital clues to the mechanisms of the disease, as the risk of developing the disease was up to $80 \%$ in sibling [15].

Identification of a unified genetic risk marker was complicated by the fact that the frequencies of susceptible and protective alleles vary widely across different ethnic groups and geographical regions [16]. Thus, type 1 diabetes risk markers should individually selected for the target population since the efficiency of screening of various markers was highly population-determined. 
In the present study, the haplotype $\mathrm{DRB} 1^{\star} 03: 01$-DQA $1^{\star} 05$ :01$\mathrm{DQB1}^{\star} 02: 01$ was significantly higher among sibling $(\mathrm{OR}=4.47)$ compared to controls. A similar association was established before in T1DM Saudi patients as compared to controls [17]. The risk of DRB1 ${ }^{\star} 03: 01-D Q A 1^{\star} 05: 01-D Q B 1{ }^{*} 02: 01$ in the homozygote form was remarkably high $(\mathrm{OR}=14)$ among Saudi siblings and compares favorably with similar previously reported frequency in Sardinian patients [12]. On the other hand, $\mathrm{DRB} 1^{\star} 03: 01-\mathrm{DQA} 1^{\star} 05: 01$ DQB $1^{\star} 02: 01$ was considered a significant risk marker among other Arabs in Bahrain and Lebanon [18]

It has been reported that individuals with two DQB1 (non-Asp) alleles and two DQA1 (Arg) alleles had the highest relative risk for disease, and both were detected in approximately $40 \%$ of IDDM patient [19]. Additionally, Dorman and Bunker [20], found that out of the four DQA1 with Arg-52 alleles (DQA1 ${ }^{\star} 03: 01 ;{ }^{*} 04: 01 ;{ }^{\star} 05: 01,{ }^{\star} 06: 01$ ), only DQA $1^{\star} 03: 01$ gave a significant contributing independent effect on T1DM risk. In many populations, DQA $1^{\star} 05: 01$ was also associated with T1DM, and this association was enhanced by the fact that $\mathrm{DQA}^{\star} 05: 01$ was usually inherited with $\mathrm{DRB} 1^{\star} 03: 01$ and $\mathrm{DQB1} 1^{\star} 02: 01$ [21].

Although DRB1 ${ }^{\star} 04$ was not significantly higher in siblings than controls, some of its subtypes showed a significant difference from controls; for example, DRB1 ${ }^{\star}$ 04:01 was significantly higher in siblings compared to controls, and it has already been established as a marker in Saudi T1DM patients [17] as well as in Belgian [22] and American patients [23]. It is also known that the DRB $1^{\star} 04: 03$ confers protection against the disease as it found to be higher in controls than siblings; similar result was reported in a previous study on Saudi patients [17]. In the current study the DRB $1^{\star} 04: 05$ was neutral in siblings, and this finding contrasts the previous report among Saudi T1DM in whom $\mathrm{DRB1}^{\star} 04: 05$ was significantly higher in T1DM patients compared to controls [17].

It is also of interest to add that the positivity to GAD and IA-2 autoantibodies gives satisfactory predictive markers for T1DM in siblings, and even in the general population [24]. Also in the Karlsburge [25] study, from Germany suggested that the combined screening of GADA and IA-2A was enough for the identification of all children at risk for the disease. In support of this, Mrena et al., [7] from Finland emphasized that IA- 2 by itself can be a predictive marker in first-degree relatives. Similarly, Decochez et al. [5] from Belgium concluded that IA-2 conferred the highest 5 -year diabetes risk $(>50 \%)$ compared to other autoantibodies (ICA, IAA, GADA), irrespective of the number of autoantibodies present.

The ethnic variation in the prevalence of autoantibodies among T1DM was highlighted in many early studies [26,27]. In the current study, GAD antibody and anti-IA-2 were significantly higher in siblings compared to controls. Similar results of antibodies prevalence were reported in the first-degree relatives from Brazil [28]. In agreement with a report from Finland [29], which found that, GAD antibodies were prevalent more in siblings than ant-IA-2.

Among Saudi nondiabetic siblings, 5.6\% were positive for both GADA and IA-2 antibodies. Similar results were reported by Pastore et al. [30], who found $4 \%$ of siblings of T1DM patients in Italy were positive for two or more antibodies. Another study considered the frequency of multiple autoantibodies was a more significant marker in disease prediction than the presence of each autoantibody alone [31].

In the present study, when correlating frequencies of antibodies with age, GAD antibody was found to be significantly higher in the age group 5 to 10 years $(50 \%)$, while all IA-2 positive were under the age of 5years (100\%), and this is in line with an earlier report by Kupila A et al. [32].

In reviewing HLA sharing between siblings and proband, we found that $27.8 \%$ of siblings were HLA-identical to the proband, $61.1 \%$ were haploidentical, and $11.1 \%$ were non-identical. A similar result was reported in a study conducted on T1DM siblings from Finland [15].

In the present study, antibodies against GADA were found to be positive in $60 \%$ of HLA-identical siblings, and $9.1 \%$ in haploidentical siblings and none of the non-identical siblings. In addition, antibodies to IA-2 were positive in $20 \%$ of HLA-identical siblings, and $9.1 \%$ of haploidentical siblings and none in non-identical HLA siblings. All siblings with multiple autoantibodies were HLA-identical with proband. Similar results were reported before among Russian [33] patients where the prevalence of GAD antibodies was significantly higher in siblings who were identical for one haplotype compared to non-identical siblings. In another Finnish study [34], autoantibody positive children within the same family carry identical HLA-DQB1 genotypes in more than $70 \%$ of the cases.

Thus the present finding of our study is that siblings of T1DM were at higher genetic risk than the normal population (controls), and this agrees with similar results from Belgians [35], and in indo-Aryan population in UK [36]. This significant association between HLA gene sharing and the appearance of autoimmune markers in siblings has also been reported among Japanese children and their families [37].

In conclusion, the results obtained in this study showed the importance of immunogenetic screening in the prediction of TIDM. The detection of $\mathrm{DRB} 1{ }^{*} 03: 01-\mathrm{DQA} 1^{\star} 05: 01-\mathrm{DQB} 1{ }^{\star} 02: 01$ in $61.1 \%$ as heterozygous and $22.2 \%$ in homozygous among siblings was considered a genetic risk marker for T1DM among Saudi first-degree siblings. Next are GAD and IA-2 autoantibodies screening which will establish the significant correlation between autoantibodies presence and HLA sharing with their proband. Thus, these findings which were report for first time among Saudi T1DM patients provide a model for establishing an early prediction marker of T1DM among sibling, which we hope will facilitate the early interventional management of the disease.

\section{Acknowledgements}

We were grateful to K. Hamam and J. Chu for their technical assistant. This research supported by a grant from Deanship of Scientific Research, King Saud University.

\section{Conflict of Interest}

We wish to confirm that there were no known conflicts of interest associated with this publication. We also confirm that the manuscript has been read and approved by all named authors and that there are no other persons who satisfied the criteria for authorship but were not listed. We further confirm that the order of authors listed in the manuscript has been approved by authors.

\section{References}

1. Skyler JS (2007) Prediction and prevention of type 1 diabetes: progress problems, and prospects. Clin Pharmacol Ther 81: 768-771.

2. Hendrieckx C, De Smet F, Kristoffersen I, Bradley C (2002) Risk assessmen for developing type 1 diabetes: intentions of behavioural changes prior to risk notification. Diabetes Metab Res Rev 18: 36-42.

3. Simonen P, Korhonen T, Simell T, Keskinen P, Kärkkäinen M, et al. (2006) Parental reactions to information about increased genetic risk of type 1 diabetes mellitus in infants. Arch Pediatr Adolesc Med 160: 1131-1136.

4. Kulmala P, Savola K, Reijonen H, Veijola R, Vähäsalo $P$, et al. (2000) Genetic markers, humoral autoimmunity, and prediction of type 1 diabetes in siblings of affected children. Childhood Diabetes in Finland Study Group. Diabetes 49 : 48-58. 
Citation: Awadalla S, AL Hakbany M (2015) HLA-DR, HLA-DQ Haplotypes and Diabetic Autoantibodies in Nondiabetic Siblings of Type I Diabetes. J Diabetes Metab 6: 580. doi:10.4172/2155-6156.1000580

5. Decochez K, Truyen I, van der Auwera B, Weets I, Vandemeulebroucke E, et al. (2005) Combined positivity for HLA DQ2/DQ8 and IA-2 antibodies defines population at high risk of developing type 1 diabetes. Diabetologia 48: 687-694.

6. Buczkowska EO, Chobot PJ (2003) [Immune markers of type-I diabetes mellitus in children with diabetes in their first-degree relatives. Part I]. Przegl Lek 60: 580-584.

7. Mrena S, Virtanen SM, Laippala P, Kulmala P, Hannila ML, et al. (2006) Models for predicting type 1 diabetes in siblings of affected children. Diabetes Care 29: 662-667.

8. Mrena S, Savola K, Kulmala P, Reijonen H, llonen J, et al. (2003) Genetic modification of risk assessment based on staging of preclinical type 1 diabetes in siblings of affected children. J Clin Endocrinol Metab 88: 2682-2689.

9. Mychaleckyj JC, Noble JA, Moonsamy PV, Carlson JA, Varney MD, et al. (2010) HLA genotyping in the international Type 1 Diabetes Genetics Consortium. Clin Trials 7: S75-87.

10. Bugawan TL, Klitz W, Alejandrino M, Ching J, Panelo A, et al. (2002) The association of specific HLA class I and II alleles with type 1 diabetes among Filipinos. Tissue Antigens 59: 452-469.

11. Sheehy MJ, Scharf SJ, Rowe JR, Neme de Gimenez MH, Meske LM, et al. (1989) A diabetes-susceptible HLA haplotype is best defined by a combination of HLA-DR and -DQ alleles. J Clin Invest 83: 830-835.

12. Cucca F, Muntoni F, Lampis R, Frau F, Argiolas L, et al. (1993) Combinations of specific DRB1, DQA1, DQB1 haplotypes are associated with insulin-dependent diabetes mellitus in Sardinia. Hum Immunol 37: 85-94.

13. Lampasona V1 Belloni C, Piquer S, Bonicchio S, Furlan R, et al. (2008) Radiobinding assay for detecting autoantibodies to single epitopes. J Immuno Methods 336: 127-134.

14. Bland JM, Altman DG (2000) Statistics notes. The odds ratio. BMJ 320: 1468.

15. Aly TA, Ide A, Jahromi MM, Barker JM, Fernando MS, et al. (2006) Extreme genetic risk for type 1A diabetes. Proc Natl Acad Sci U S A 103: 14074-14079.

16. Steenkiste A, Valdes AM, Feolo M, Hoffman D, Concannon P, et al. (2007) 14th International HLA and Immunogenetics Workshop: report on the HLA component of type 1 diabetes. Tissue Antigens 69 Suppl 1: 214-225

17. Manan H, Angham AM, Sitelbanat A (2010) Genetic and diabetic auto-antibody markers in Saudi children with type 1 diabetes. Hum Immunol 71: 1238-1242.

18. Al-Jenaidi FA, Wakim-Ghorayeb SF, Al-Abbasi A, Arekat MR, Irani-Hakime N et al. (2005) Contribution of selective HLA-DRB1/DQB1 alleles and haplotypes to the genetic susceptibility of type 1 diabetes among Lebanese and Bahrain Arabs. J Clin Endocrinol Metab 90: 5104-5109.

19. Tosi G, Facchin A, Pinelli L, Accolla RS (1994) Assessment of the DQB1-DQA1 complete genotype allows best prediction for IDDM. Diabetes Care 17: 10451049 .

20. Dorman JS, Bunker CH (2000) HLA-DQ locus of the human leukocyte antigen complex and type 1 diabetes mellitus: a HuGE review. Epidemiol Rev 22: 218227.

21. Erlich H, Valdes AM, Noble J, Carlson JA, Varney M, et al. (2008) HLA DR$\mathrm{DQ}$ haplotypes and genotypes and type 1 diabetes risk: analysis of the type 1 diabetes genetics consortium families. Diabetes 57: 1084-1092.

22. Donner H, Seidl C, Van der Auwera B, Braun J, Siegmund T, et al. (2000) HLADRB1*04 and susceptibility to type 1 diabetes mellitus in a German/Belgian family and German case-control study. The Belgian Diabetes Registry. Tissue Antigens 55: 271-274.

23. Thomson G, Valdes AM, Noble JA, Kockum I, Grote MN, et al. (2007) Relative predispositional effects of HLA class II DRB1-DQB1 haplotypes and genotypes on type 1 diabetes: a meta-analysis. Tissue Antigens 70: 110-127.
24. De Grijse J, Asanghanwa M, Nouthe B, Albrecher N, Goubert P, et al. (2010) Predictive power of screening for antibodies against insulinoma-associated protein 2 beta (IA-2beta) and zinc transporter-8 to select first-degree relatives of type 1 diabetic patients with risk of rapid progression to clinical onset of the disease: implications for prevention trials. Diabetologia 53: 517-524.

25. Sosenko JM, Krischer JP, Palmer JP, Mahon J, Cowie C, et al. (2008) A risk score for type 1 diabetes derived from autoantibody-positive participants in the diabetes prevention trial-type 1. Diabetes Care 31: 528-533.

26. Wang J, Miao D, Wang Y, Lu B, Babu S, et al. (2011) Analysis of pathogenesis of juvenile new-onset diabetes. J Diabetes 3: 132-137.

27. Brandao LC, Vatta S, Guimaraes R, Segat L, Araujo J, et al. (2010) Rapid genetic screening for major human leukocyte antigen risk haplotypes in patients with type 1 diabetes from Northeastern Brazil. Hum Immunol 71: 277-280.

28. Pardini VC, Mourao DM, Nascimento PD, Vívolo MA, Ferreira SR, et al. (1999) Frequency of islet cell autoantibodies (IA-2 and GAD) in young Brazilian type 1 diabetes patients. Braz J Med Biol Res 32: 1195-1198.

29. Siljander HT, Veijola R, Reunanen A, Virtanen SM, Akerblom HK, et al. (2007) Prediction of type 1 diabetes among siblings of affected children and in the general population. Diabetologia 50: 2272-2275.

30. Pastore MR, Bazzigaluppi E, Bonfanti R, Dozio N, Sergi A, et al. (1998) Two step islet autoantibody screening for risk assessment of type 1 diabetes in relatives. Diabetes Care 21: 1445-1450.

31. Eisenbarth GS, Jeffrey J (2008) The natural history of type 1A diabetes. Arq Bras Endocrinol Metabol 52: 146-155.

32. Kupila A, Keskinen P, Simell T, Erkkilä S, Arvilommi P, et al. (2002) Genetic risk determines the emergence of diabetes-associated autoantibodies in young children. Diabetes 51: 646-651.

33. Kulaeva TL, Titovich EV, Zil'berman LI, Nosikov VV, Peterkova VA, et al. (2003) [Genetic and immunologic aspects of type 1 diabetes mellitus]. Usp Fiziol Nauk 34: 45-62.

34. Veijola $R$, Vahasalo $P$, Tuomilehto-Wolf $E$, Reijonen $H$, Kulmala $P$, et al. (1995) Human leukocyte antigen identity and DQ risk alleles in autoantibodypositive siblings of children with IDDM are associated with reduced early insulin response. Childhood Diabetes in Finland (DiMe) Study Group. Diabetes. 44: 1021-8.

35. Vandewalle CL, Falorni A, Lernmark A, Goubert P, Dorchy H, et al. (1997) Associations of GAD65- and IA-2- autoantibodies with genetic risk markers in new-onset IDDM patients and their siblings. The Belgian Diabetes Registry. Diabetes Care 20: 1547-1552.

36. Kelly MA, Alvi NS, Croft NJ, Mijovic CH, Bottazzo GF, et al. (2000) Genetic and immunological characteristics of Type I diabetes mellitus in an Indo-Aryan population. Diabetologia 43: 450-456.

37. Sugihara S, Ogata T, Kawamura T, Urakami T, Takemoto K, et al. (2012) HLAclass II and class I genotypes among Japanese children with Type 1A diabetes and their families. Pediatr Diabetes 13: 33-44. 